\title{
Selected mucolytic, anti-inflammatory and cardiovascular drugs change the ability of neutrophils to form extracellular traps (NETs)*
}

\author{
Marcin Zawrotniak, Andrzej Kozik and Maria Rapala-Kozik ${ }^{\bowtie}$ \\ Department of Analytical Biochemistry, Faculty of Biochemistry, Biophysics and Biotechnology, Jagiellonian University in Kraków, Kraków, Poland
}

\begin{abstract}
Neutrophils form the first line of host defense against infections that combat pathogens using two major mechanisms, the phagocytosis or the release of neutrophil extracellular traps (NETs). The netosis (NET formation) exerts additional, unfavorable effects on the fitness of host cells and is also involved at the sites of lung infection, increasing the mucus viscosity and in the circulatory system where it can influence the intravascular clot formation. Although molecular mechanisms underlying the netosis are still incompletely understood, a role of NADPH oxidase that activates the production of reactive oxygen species (ROS) during the initiation of NETs has been well documented. Since several commonly used drugs can affects the netosis, our current study was aimed to determine the effects of selected mucolytic, anti-inflammatory and cardiovascular drugs on NET formation, with a special emphasis on ROS production and NADPH oxidase activity. The treatment of neutrophils with $\mathrm{N}$-acetylcysteine, ketoprofen and ethamsylate reduced the production of ROS by these cells in a dosedependent manner. NET formation was also modulated by selected drugs. $\mathrm{N}$-acetylcysteine inhibited the netosis but in the presence of $\mathrm{H}_{2} \mathrm{O}_{2}$ this neutrophil ability was restored, indicating that $\mathrm{N}$-acetylcysteine may influence the NET formation by modulating ROS productivity. The administration of ethamsylate led to a significant reduction in NET formation and this effect was not restored by $\mathrm{H}_{2} \mathrm{O}_{2}$ or $\mathrm{S}$. aureus, suggesting the unexpected additional side effects of this drug. Ketoprofen seemed to promote ROS-independent NET release, simultaneously inhibiting ROS production. The results, obtained in this study strongly suggest that the therapeutic strategies applied in many neutrophil-mediated diseases should take into account the NET-associated effects.
\end{abstract}

Key words: neutrophil extracellular trap, reactive oxygen species, inflammation

Received: 9 April, 2015; revised: 02 May, 2015; accepted: 21 May, 2015; available on-line: 20 August, 2015

\section{INTRODUCTION}

Neutrophils are the immune cells of primary importance for the host defense system. During the infections, neutrophils leave the blood vessels and are recruited to the site of inflammation, where they kill pathogens using several mechanisms such as the phagocytosis, degranulation or releasing neutrophil extracellular traps (NETs), the latter process also known as netosis (Kumar \& Sharma, 2010). NETs seem to be specially designed to catch and kill pathogens outside the cell. They are composed of decondensed chromatin, decorated with many antimicrobial factors released from internal neutrophil granules. Nuclear DNA fibers form a trap scaffold responsible for keeping all antimicrobial factors at the place of infection and providing their high local concentration. Moreover, though trapping microbial cells, these prevent the further spreading of microorganisms over the host organism (Brinkmann et al., 2004). Antimicrobial activity of NETs is determined by a number of antibacterial factors, originally stored in neutrophil granules. Many of these factors are proteins with antimicrobial activity such as elastase, cathepsin $\mathrm{G}$, proteinase 3, lactoferrin and myeloperoxidase (MPO) as well as short antimicrobial peptides, e.g., LL-37 cathelicidin (Urban et al., 2009). All of them can inactivate and kill pathogens by neutralizing their virulence factors or disintegrating the pathogen cell membrane (Brinkmann \& Zychlinsky, 2012).

A large number of stimuli activate neutrophils to release NETs. The netosis can be triggered by chemical factors such as phorbol-12-myristate-13-acetate (PMA), lipopolysaccharide (LPS) and hydrogen peroxide as well as by microorganisms, e.g., Escherichia coli, Klebsiella pneumoniae, Staphylococcus aureus, Candida albicans (Guimarães-Costa et al., 2012). The mechanism of NETs release is incompletely understood, but it has been well established that at least two different ways of NET formation are possible, either dependent or independent on the reactive oxygen species (ROS) production (Nishinaka et al., 2011; Kaplan \& Radic, 2012). The type of netosis is determined by the activating factors, especially the type of the encountered microorganisms. In the ROS-dependent pathway, a key step is the activation of NADPH oxidase (PHOX) that responds with a massive production of ROS (Parker et al., 2012). Neutrophils isolated from patients with chronic granulomatous disease (CGD), in which mutations in PHOX subunits suppress the enzyme activity and, hence, the ROS production cannot release NETs. However, after treatment of the

e-mail: maria.rapala-kozik@uj.edu.pl

* Preliminary report on the same subject has been presented during the 42nd Winter School of Faculty of Biochemistry, Biophysics and Biotechnology, Zakopane 10-14 February 2015.

Abbreviations: CGD, chronic granulomatous disease; DMSO, dimethylsulfoxide; DNase, Deoxyribonuclease I; DPI, diphenyleneiodonium chloride; LPS, lipopolysaccharide; MNase, micrococcal nuclease; $\mathrm{MOI}$, at a multiplicity of infection; MPO, myeloperoxidase; MTT, tetrazolium dye; NAC, N-acetylcysteine; NETs, neutrophil extracellular traps; NSAID, non-steroidal anti-inflammatory drug; PBS, phosphate buffered saline; PHOX, phagocyte NADPH oxidase; PMA, phorbol-12-myristate-13-acetate; ROS, reactive oxygen species; SLE, systemic lupus erythematosus. 
neutrophils with $\mathrm{H}_{2} \mathrm{O}_{2}$ the ability of NET production is restored. A similar effect, the netosis blocking was also observed after treatment of PHOX with diphenyleneiodonium chloride (DPI), the effective enzyme inhibitor (Li \& Trush, 1998; Nishinaka et al., 2011; Bianchi et al., 2009). On the contrary, in the ROS-independent netosis, NETs are released without PHOX activation (Douda et al., 2015). This mechanism, significantly faster than the former, was first identified in neutrophils that responded to the infections caused by Staphylococcus aureus (Pilsczek et al., 2010) and is probably mediated by mitochondrial ROS and calciumactivated small conductance potassium ion channels (Douda et al., 2015). This pathogen developed several defense mechanisms against neutrophils such as escaping from phagosomes, the inhibition of phagocytosis and neutralizing ROS (Pilsczek et al., 2010). Therefore, the external mechanism of pathogen killing, such as the NET release should focus particular attention.

Besides antimicrobial activity of NETs, beneficial for the host, these structures are also involved in some immunological diseases and other pathological processes. High NET levels were detected during chronic inflammatory, autoimmune disease (SLE), thrombosis, acute lung injury, cystic fibrosis and other (Amulic et al., 2012; Zawrotniak \& Rapala-Kozik, 2013). In most cases the presence of NETs have a negative impact on the fitness of host tissues, because the high concentration of antimicrobial agents and ineffective clearance of NETs led to a number of tissue damages at the site of NET production (Saffarzadeh et al., 2012). But in some situations NETs supports the host functions, e.g., in a bleeding, where NETs can serve as a scaffold for clot formation and help trapping the blood morphotic elements or proteins, like platelets or fibrinogen (Fuchs et al., 2010; Fuchs et al., 2012).

However, the release of NETs or ROS production by neutrophils can be modified by specific inhibitors or drugs. DPI prevents netosis on ROS-dependent pathway, DNase helps in NET clearance by degradation of their scaffold (Duranton et al., 2000; Papayannopoulos et al., 2011), acetylsalicylic acid can inhibit the MPO activity (Lapponi et al., 2013), cyclosporine A modulate calcium flux and inhibit the ROS-independent pathway of netosis (Gupta et al., 2014). Most of these compounds directly target the neutrophil activity, preventing negative impact of NET release. But commonly used drugs like statins (Chow et al., 2010), flavonoids and aminosalicylic acid (Kirchner et al., 2013) sometimes show an undesirable effects against neutrophils, switching off some helpful mechanisms of these immune cells (Lapponi et al., 2013).

Application of proteomics to the identification of NET components or microbial proteins involved in pathogen trapping by NETs suggests that a combination of proteomic approach and traditional biochemical methods can provide new insights into the role of neutrophils in immune responses. Moreover, extension of those studies on disease-related networks can improve understanding of inflammatory diseases or identify biomarkers of those diseases, and reveal new therapeutic targets (McLeish et al., 2013).

Our current study was aimed to reveal the impact of selected drugs on ability of neutrophils to release NETs, with a special focus on ROS production and PHOX activity. We examined selected mucolytic (N-acetylcysteine), anti-inflammatory (clemastine, ketoprofen, hydrocortisone) and cardiovascular (ethamsylate) drugs, commonly used in therapy of various diseases.

\section{MATERIAL AND METHODS}

Clemastine was purchased from Polfa (Warszawa, Poland); Ethamsylate was from Galena (Wrocław, Poland); Hydrocortisone was from Jelfa (Jelenia Góra, Poland); Ketoprofen was from Lek S.A. (Stryków, Poland); Luminol, N-acetylcysteine, Polyvinyl alcohol, Tetrazolium dye (MTT) were from Sigma-Aldrich (Saint Louis, USA); Micrococcal nuclease was from Roche (Penzberg, Germany); Pancoll $1.077 \mathrm{~g} / 1$ was from PAN-Biotech (Aidenbach, Germany); Sytox Green was from Molecular Probes (Eugene, USA).

Isolation of neutrophils from human peripheral blood. Human neutrophils were isolated from EDTAtreated whole blood obtained from healthy donors, supplied by the Regional Blood Donation Center, Kraków, Poland. The neutrophil-containing fraction was collected by Pancoll $1.077 \mathrm{~g} / 1$ gradient separation, performed according to the manufacturer's protocols. The high-density fraction, containing neutrophils and erythrocytes, were mixed with a solution of polyvinyl alcohol (1\%) and incubated for $20 \mathrm{~min}$ at room temperature. The upper layer was collected and centrifuged. The cell pellet was transferred to hypotonic solution to remove erythrocytes contamination by their lysis. After washing with phosphate buffered saline (PBS) the cells were resuspended in RPMI 1640 medium (phenol-red free). The neutrophil purity was typically $>95 \%$, as assessed by forward-scatter and side-scatter flow cytometric analyses.

Neutrophil viability assay. Neutrophils $\left(2 \times 10^{5}\right.$ cells/well) were suspended in RPMI medium in the wells of a 96-well microplate, treated with various concentrations of selected drugs for 30 minutes and washed 3 times with PBS. Then, the cells were incubated with MTT solution $(0.5 \mathrm{mg} / \mathrm{ml})$ for 2 hours. The purple precipitate was solubilized with dimethylsulfoxide (DMSO) and absorbance at $560 \mathrm{~nm}$ was measured using a BioTek Synergy H1 microplate reader (BioTek, Winooski, USA).

Neutrophils $\left(2.2 \times 10^{5}\right.$ cells per well), pre-treated for 30 minutes with various concentrations of selected drugs and washed 3 times with PBS, were allowed to settle in the wells of 96 -well white microplate, at $37^{\circ} \mathrm{C}$ for $30 \mathrm{~min}$, in $160 \mu \mathrm{l}$ of Krebs-Ringer phosphate buffer containing freshly prepared luminol solution $\left(10^{-6} \mathrm{M}\right)$. As a control, the untreated cells were used. The neutrophils were then stimulated with $20 \mu \mathrm{l}$ of $25 \mathrm{nM}$ PMA or PBS (as a negative control). The chemiluminescence was recorded immediately after PMA application for one hour, using a BioTek Synergy H1 microplate reader, with onesecond integration time.

NET visualization and quantification. The 12 -well microplates (Greiner Bio-One, Germany) were coated with $0.01 \%$ poly-L-lysine in RPMI at $4^{\circ} \mathrm{C}$ overnight. After drying, $1.5 \times 10^{6}$ neutrophils suspended in $900 \mu \mathrm{l}$ RPMI were seeded into each well. The cells were incubated for 30 minutes at $37^{\circ} \mathrm{C}$, and then the attached neutrophils were treated with specified concentrations of each tested drugs for 30 minutes. After washing with PBS, the cells were stimulated at $37^{\circ} \mathrm{C}$ for 3 hours with various factors: $25 \mathrm{nM}$ PMA, $5 \mu \mathrm{M}$ DPI, $0.03 \% \mathrm{H}_{2} \mathrm{O}_{2}$ or $S$. aureus at a multiplicity of infection (MOI) of 10 .

Visualisation. After incubation, Sytox Green dye was added to each well at the final concentration of $1 \mu \mathrm{M}$. NETs were observed in a fluorescence microscope Nikon Eclipse Ti (Nikon Instruments, Melville, USA).

Quantification. After incubation, the wells were washed 3 times with PBS, then $400 \mu$ l of micrococcal nuclease $(1 \mathrm{U} / \mathrm{ml})$ were added and the microplates were incubated at $37^{\circ} \mathrm{C}$ for further 20 minutes. The 
enzymatic reaction was stopped with EDTA $(100 \mu \mathrm{g} /$ $\mathrm{ml})$, the solution was collected and Sytox Green at the final concentration $1 \mu \mathrm{M}$ was added. Portions $(50 \mu \mathrm{l})$ of each sample were transferred to 96-well microplates and fluorescence was measured using the Biotek Synergy H1 microplate reader at excitation $465 \mathrm{~nm}$ and emission $525 \mathrm{~nm}$.

\section{RESULTS}

\section{All drugs tested in this study do not lead to death of neutrophils in vitro}

Bearing in mind that NETs can influence mucolytic properties of lung tissues, cardiovascular conditions and inflammatory responses, for this study we chose five medicaments, commonly used both in health emergency conditions or in long-term therapy, i.e., clemastine, hydrocortisone, $\mathrm{N}$-acetylcysteine, ketoprofen and ethamsylate.

The concentrations of the tested drugs were used in accordance with their expected concentrations in a human serum after administration of typical therapeutic doses by injection directly to circulatory system or administered orally to adult patients. Most of these values are specified in literature (Regenthal et al., 1999; Borgström et al., 1986; Hernandez et al., 2004), but if not, the concentrations were calculated as the ratio of the therapeutic dose administered to the average volume (6 liters) of blood in the human body.

To exclude any toxic effects of selected drugs on neutrophils, we checked the viability of the cells after treatment by selected compounds for 30 minutes, in a wide range of drug concentrations. As a negative control the untreated cells were used, and for a positive control the cells were treated with $25 \mathrm{nM}$ PMA.

The viability was tested using MT'T test, in which the reduction of tetrazolium dye to purple formazan was monitored at $560 \mathrm{~nm}$. Viable cells performed the reaction efficiently and the yield of the reaction indicated the state of viability, while a decrease in the amount of the product suggested cell death. None of tested drugs led to neutrophil death under tested conditions (Fig. 1). Except for ethamsylate, the p-value for the comparison of the samples versus the negative control $>0.1$, suggesting that the differences were insignificant. The $p$-value for ethamsylate was $* p<0.05$, but the absorbance level for the drug was higher than for negative control, suggesting the effect of prolonged lifetime of neutrophils. Positive control showed highly increased cell death, which is associated with the activation of netosis pathway.

\section{ROS production by PMA stimulated neutrophils is inhibited by $\mathrm{N}$-acetylcysteine, ketoprofen and ethamsylate}

Neutrophils produce high amounts of ROS, serving as antimicrobial factors or signaling molecules. The mechanism of ROS production that depends on the activity of PHOX is highly efficient and very fast. The activity of this enzyme can be modified by many compounds, causing dramatic changes in physiology and immune response of neutrophils. A chemical agent, PMA, potently activates neutrophils leading to production of ROS in few minutes, and in consequence to NET release.

We checked the effect of neutrophil pretreatment with tested drugs on the ability of the cells to produce ROS in a response to further PMA stimulation. All drugs

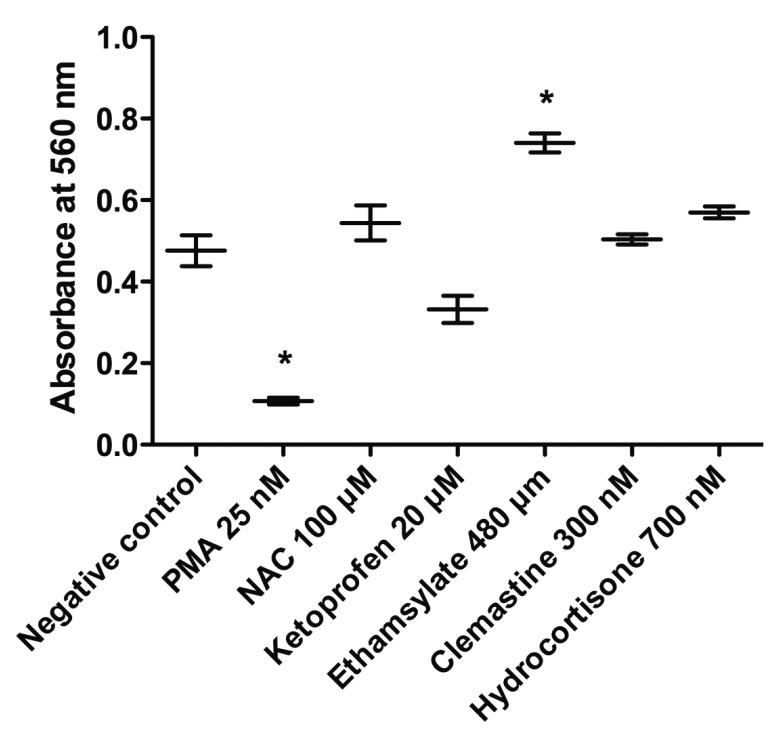

Figure 1. The viability of neutrophils in the presence of tested drugs, determined by MTT assay.

Neutrophils $\left(2 \times 10^{5}\right.$ cells/well) were suspended in RPMI medium and treated with selected concentrations of NAC, ketoprofen, ethamsylate, clemastine and hydrocortisone for 30 minutes. Then, neutrophils were incubated with solution of MTT dye $(0.5 \mathrm{mg} / \mathrm{ml})$ for 2 hours and the absorbance at $560 \mathrm{~nm}$ was measured. Data represent mean values of absorbance from two replicates \pm S.D. Asterisks denote differences considered as statistically significant at $p<0.05$ as compared to the negative control (untreated cells) by one-way ANOVA with Dunnett's multiple comparison test.

were tested at a broad range of concentrations, covering their concentrations in serum after administration of therapeutic dosages.

The obtained results are presented as the percentage ratio of maximal luminescence signal from drug-treated cells to untreated cells, stimulated by PMA (Fig. 2). Luminescence of negative control (cells not treated by any chemicals) showed a very low level of spontaneous ROS release by neutrophils. The samples pretreated with PHOX inhibitor (DPI) presented only $10 \%$ of total ROS production by neutrophils with active PHOX.

$\mathrm{N}$-acetylcysteine (NAC), ketoprofen and ethamsylate caused a significant and dose-dependent inhibition of ROS generation. At the concentrations that correspond to the therapeutic doses, NAC, ketoprofen and ethamsylate decreased the ROS production by about $30 \%$ (Fig. 2A-C). Higher concentrations of these drugs led to a further reduction of ROS amount to the level that corresponded to DPI action.

On the contrary, clemastine and hydrocortisone exerted slight effects on ROS generation by neutrophils under PMA treatment, suggesting no influence on PHOX activity in vitro. Only at the highest concentration of clemastine a rapid decrease of ROS release was observed, but it was probably caused by overdose and cell death.

\section{Release of NETs is modulated by N-acetylcysteine, ketoprofen and ethamsylate}

Because the release of NETs by neutrophils depends on various factors, such as stimulant nature, cell fitness and the type of netosis-activation pathway, we checked the influence of pretreatment of neutrophils with tested drugs on the subsequent netosis process. Given results presented (Fig. 3A), some of tested drugs modified the neutrophils response as the NET release. Neutrophils pretreated with $0.03 \mu \mathrm{M}$ clem- 
astine and $0.7 \mu \mathrm{M}$ hydrocortisone, followed by the stimulation with PMA, produced NETs at the same the negative control. However, on Fig. 3B the dosedependent inhibition of NETs release by neutrophils
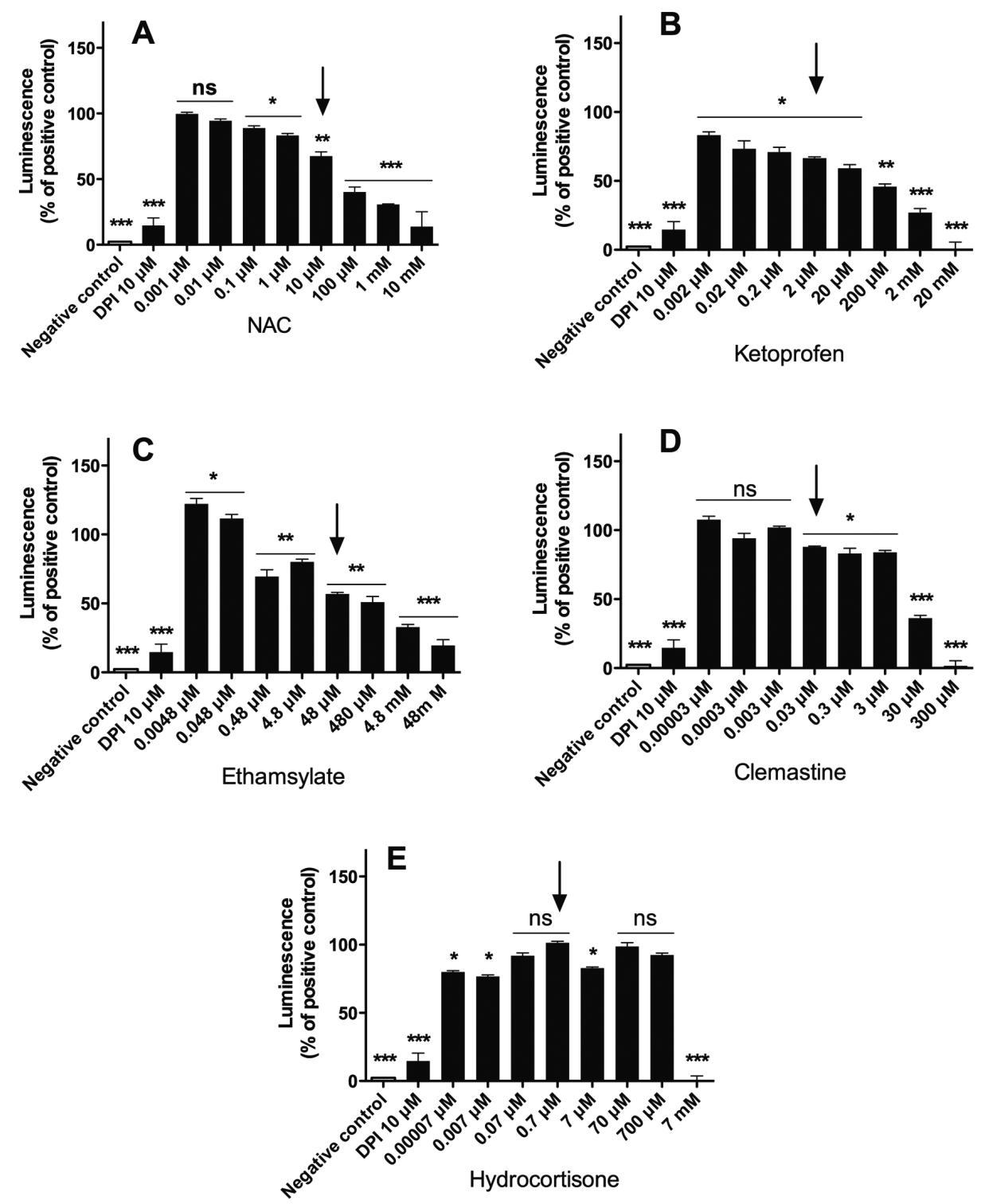

Figure 2. Luminescence assay of ROS production by neutrophils treated with tested drugs.

Neutrophils $\left(2 \times 10^{5}\right.$ cells/well) were suspended in RPMI medium and treated with selected concentrations of NAC (A), ketoprofen (B), ethamsylate (C), clemastine (D) and hydrocortisone (E) or $10 \mu \mathrm{M}$ DPI for 30 minutes. After washing and re-suspension in a luminol solution, the cells were stimulated with $25 \mathrm{nM}$ PMA and thereafter the luminescence was measured for $1 \mathrm{~h}$. The arrows point out the expected concentration of the drugs in the serum. Data presented the values of maximum luminescence \pm S.D. from two replicates as the percentage ratio of maximum luminescence of drug-treated neutrophils to untreated cells. Asterisks denote differences considered as statistically significant at $P<0.05(A, B, C)$. No significant differences were marked as "ns".

level as the positive control, without any differences in amount, shapes and surface area of released DNA. Moreover, the results were confirmed by the quantification of extracellular DNA released by neutrophils pretreated with the drugs at the wide concentration range (Fig. 3B). It suggests that these two drug types have no impact on netosis process regardless of its mechanism, whether ROS-dependent or ROS-independent. On the other hand, $10 \mu \mathrm{M}$ NAC and $48 \mu \mathrm{M}$ ethamsylate (Fig. 3A) caused a high decrease in NET production by neutrophils. After a half-hour treatment with therapeutic doses of drugs, the number of neutrophils that released traps was significantly lower than in the positive control, but still higher than in was observed. The fluorescence intensity detected with $100 \mu \mathrm{M}$ NAC dropped to $30 \%$ of positive control. Similarly, with $480 \mu \mathrm{M}$ ethamsylate only $40 \%$ of fluorescence was measured (Fig. 3B).

A quite different effect was observed for neutrophils treated with $20 \mu \mathrm{M}$ ketoprofen. In contrast to results described above, ketoprofen did not cause any inhibition of netosis, but instead significantly increased release of the NETs, in comparison to positive control (Fig. 3A). The results were confirmed by dose-dependent correlation (Fig. 3B); for $20 \mu \mathrm{M}$ and $200 \mu \mathrm{M}$ ketoprofen the amount of released NETs increased up to $140 \%$ and $160 \%$ of the positive control, respectively. 
A

negative

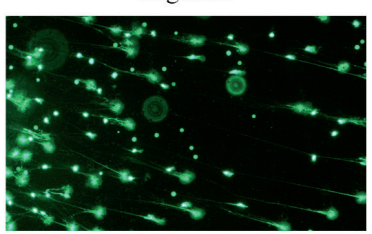

positive (25 nM PMA)

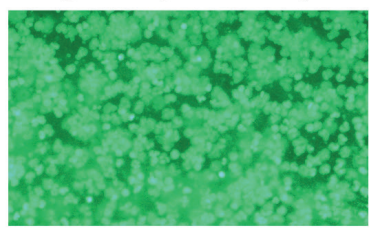

B
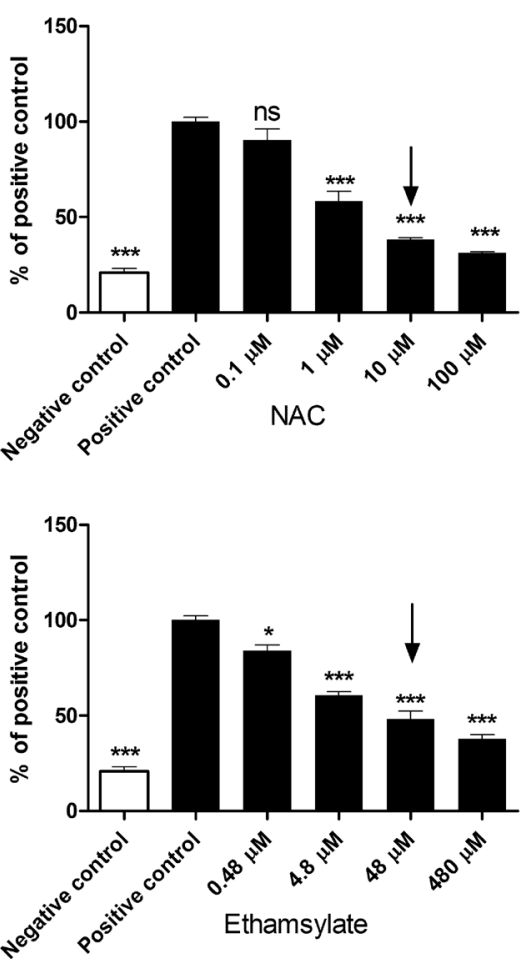

ketoprofen $(20 \mathrm{uM})$

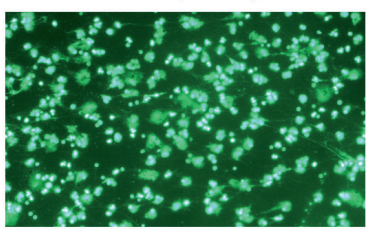

clemastine $(0.03 \mathrm{uM})$

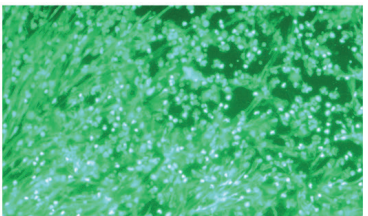

ethamsylate $(48 \mathrm{uM})$

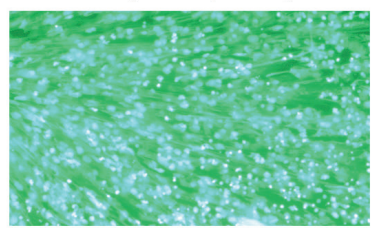

hydrocortisone $(0.7 \mathrm{uM})$

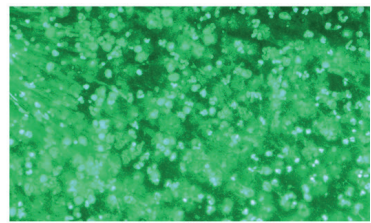

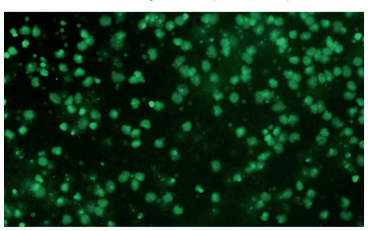
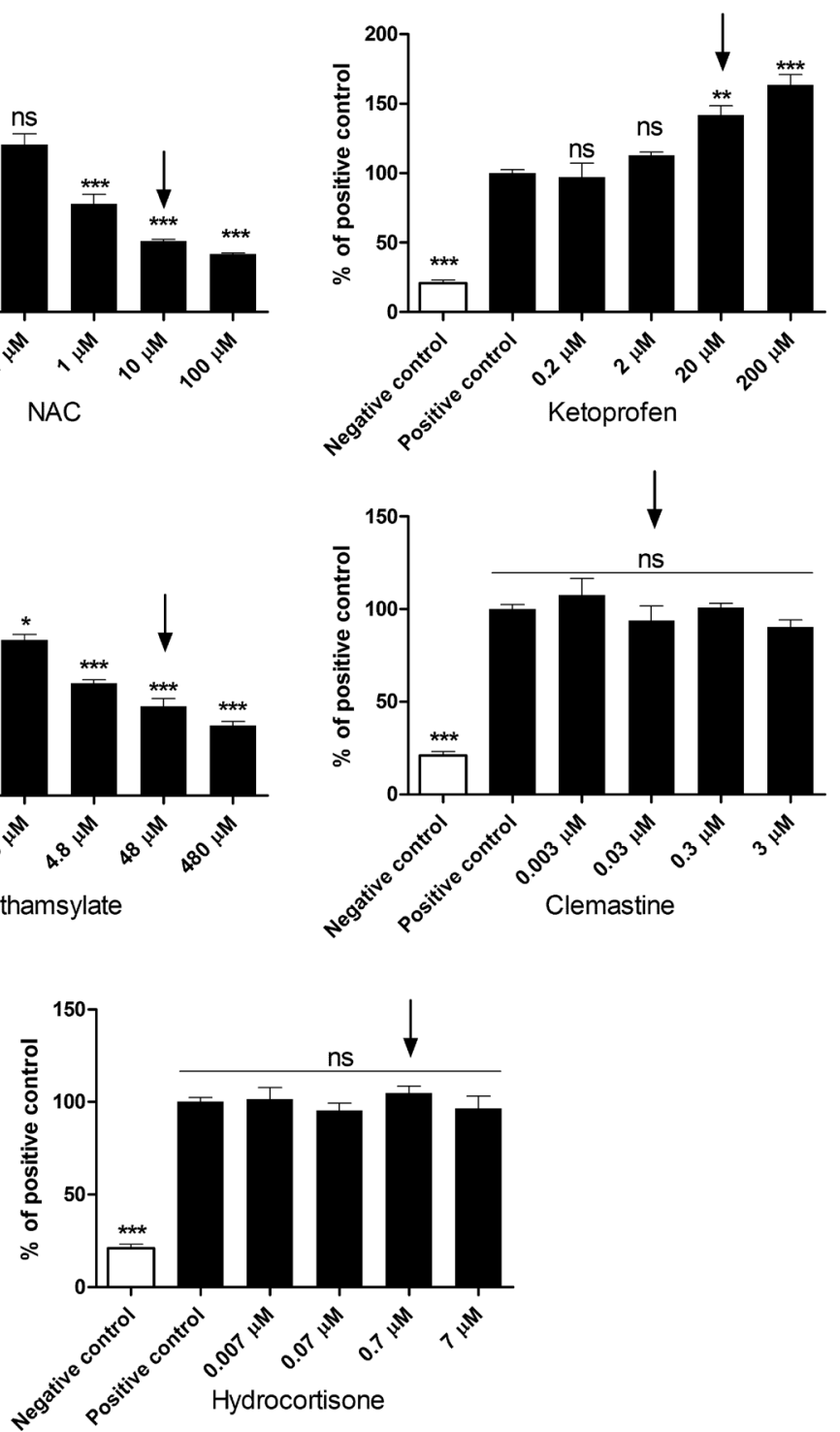

Figure 3. Effect of selected drugs on the ability of neutrophils to release NETs.

Isolated neutrophils, transferred into 12 -wells microplate $\left(1.5 \times 10^{6}\right.$ cells/well) were treated with specified concentrations of each tested drugs for 30 minutes. After washing, the neutrophils were stimulated for 3 hours with 25 nM PMA. For negative and positive control the cells were not treated with any drugs; for the negative control the cells were also not stimulated with PMA. (A) Extracellular DNA was stained with Sytox Green and visualized in fluorescence microscope. (B) Partially digested DNA was collected from each well after micrococcal nuclease treatment and quantified at presence of Sytox Green by fluorescence measurement. Data presented the values \pm S.D. from two replicates as the percentage ratio versus positive control. Differences were considered statistically significant at $P<0.05$ as compared to the positive control by one-way ANOVA with Dunnett's multiple comparison test. At the figures, significant differences were marked with asterisks $\left({ }^{*} P<0.05,{ }^{* *} P<0.01\right.$, $\left.{ }^{* * *} P<0.001\right)$. 

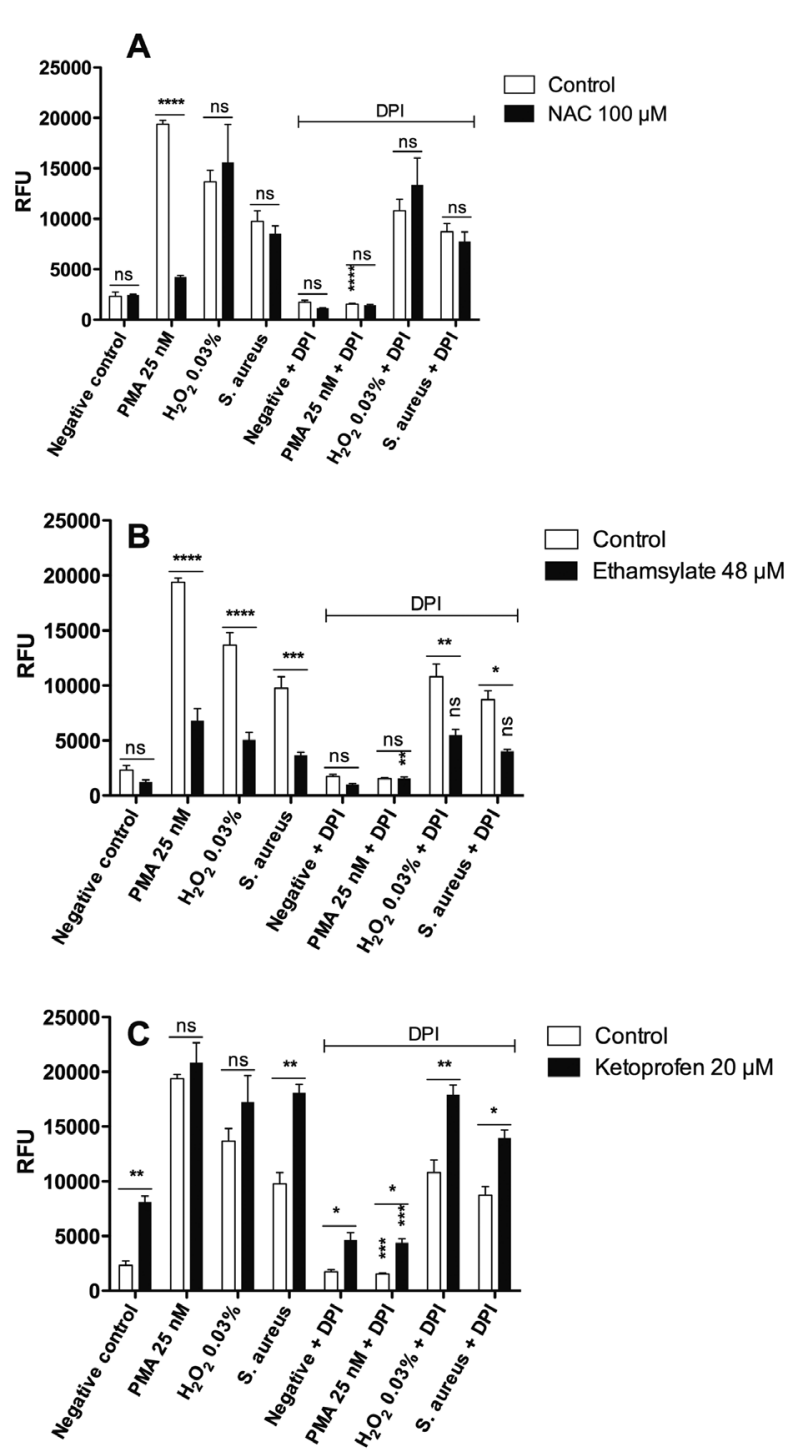

Figure 4. NET release by neutrophils in response to NAC, ethamsylate and ketoprofen.

Neutrophils $\left(1.5 \times 10^{6}\right.$ cells/well) were treated with specified concentrations of the drugs for 30 minutes. After washing, the neutrophils were stimulated for 3 hours with different stimulants: 25 nM PMA, $0.03 \% \mathrm{H}_{2} \mathrm{O}_{2}$ or S. aureus (MOI 10). Control cells (open bars) were not treated with drugs. A $5 \mu \mathrm{M}$ DPI was used to examine the ROS-independent netosis pathway. Partially digested DNA was collected from each well after micrococcal nuclease treatment and quantified as it was presented above.

\section{The mechanisms of $\mathrm{N}$-acetylcysteine, ethamsylate and ketoprofen influence on NET formation by neutrophils are different}

Taking into account the observed effects of selected drugs on the ability of neutrophils to release NETs, we further examined in details this process, considering two different, ROS-dependent and ROS-independent pathways. We focused on three of examined drugs - NAC, ethamsylate and ketoprofen - because only those medicaments showed a significant effect on neutrophils. The neutrophils were treated with a therapeutic concentration of each drug as previously but their further stimulation toward NET release was performed using different stimulants. PMA is able to trigger ROS-dependent
NET formation through the protein kinase C (PKC)dependent signaling pathway (Keshari et al., 2012). DPI, the inhibitor of PHOX, switches off the ROS-dependent netosis pathway by blocking ROS production. Hydrogen peroxide allows to produce NETs by neutrophils, bypassing NADPH-oxidase activity (Nishinaka et al., 2011). The ROS-independent netosis pathway is triggered by activation of neutrophils upon the contact with $S$. aureus (Pilsczek et al., 2010). Application of the stimulants during neutrophils treatment with above selected drugs can help in identification of the mechanisms, which were affected in neutrophils by these medicaments. As presented on Fig. 4A, the treatment of neutrophils with N-acetylcysteine influenced the NET production on the ROSdependent process, mediated by PHOX. The amount of DNA released to extracellular space in response to PMA treatment was more than four times lower for neutrophils treated with NAC as compared to untreated cells. After inhibition of PHOX with DPI, the amount of NETs for treated and for control samples dropped to the level of the negative control. The NET release during cell treatment with $0.03 \% \mathrm{H}_{2} \mathrm{O}_{2}$ was not influenced by NAC. This drug also exerted no effect on ROS-independent netosis pathway, because cells treated and untreated with NAC released the same level of DNA in response to the contact with $S$. aureus cells. In this case, DPI had no effect on neutrophil ability to form NETs.

The effect of ethamsylate on netosis pathway seemed to be more complex. A significant inhibition of NET formation by neutrophils treated with ethamsylate was observed as regards the ROS-dependent netosis pathway, after stimulation with PMA (Fig. 4B). The amount of released NETs was three times lower in cells under drug presence than in control cells. DPI blocked this part of NET formation. Stimulation of neutrophils with $0.03 \% \mathrm{H}_{2} \mathrm{O}_{2}$ have no effect on restoration of the ability of drug-treated neutrophils to release NETs. The partial effect was observed as regards the ROS-independent pathway. Stimulation of neutrophils with $S$. aureus gave three times lower amount of released DNA. It is possible that ethamsylate can act on the common part of ROS-dependent and ROS-independent netosis pathways, causing a significant decrease in NET formation.

The action of ketoprofen is quite different and multifaceted. Although ketoprofen presented the ability to inhibit ROS production by PHOX (Fig. 2B), the drug was also involved in an additional effect of NET generation, probably on the ROS-independent pathway (Fig. 4C). In all treatments, neutrophils preincubated with ketoprofen exhibited increased production of NETs. Moreover, unstimulated neutrophils, treated with $20 \mu \mathrm{M}$ ketoprofen released two-fold more NETs than control cells. An activation of ROS-dependent pathway by PMA led to release of NETs by both types of cells at the same level. DPI treatment pointed additional stimulation of NETs release by ketoprofen treatment. There was also no significant difference in the amount of DNA released by neutrophils stimulated with hydrogen peroxide. In a ROS-independent pathway ketoprofen-treated neutrophils produce two-fold more NETs than untreated cells stimulated with $S$. aureus. We can speculate that ketoprofen is able to activate NET production involving different netosis pathways.

\section{DISCUSSION}

Neutrophils play an important role in keeping homeostasis of human body and preventing spreading microbial 
infections in the host. These tasks are executed using various mechanisms, one of the major being the release of NETs. The netosis is also important for many other physiological and pathological processes in the human organism (Zawrotniak \& Rapala-Kozik, 2013). This special activity of neutrophils can be modulated at different stages by various types of drugs or other chemical factors (Lapponi et al., 2013).

In the current study we examined the effects of a range of medicaments that can affect the netosis processes, proceeding through both oxidative and non-oxidative pathways. We selected five drugs commonly used in health emergency conditions or in long-term therapy.

This study helped to understand possible consequences of the patient treatment with examined drugs, as well as presented the different aspects of the drug usage, not taken into account so far.

One of the broadly used drugs is NAC. This thiol mucolytic drug exerts many different therapeutic effects on the human body. The main application concerns a support therapy in almost all respiratory diseases, such as acute respiratory distress syndrome, acute lung injury, cystic fibrosis etc. NAC was also proposed as useful drug in therapy of cancer and cardiovascular diseases. (Masha \& Martina, 2014; Wu et al., 2014; Zafarullah et al., 2003; Kharazmi et al., 1988; Holdiness, 1991). The current examination of ROS production by neutrophils treated with NAC showed that this drug decreased ROS amounts in a dose-dependent manner. This activity could be associated with NAC ability to scavenge free radicals, which was earlier presented for higher concentration of the used drug (Zafarullah et al., 2003; Kharazmi et al., 1988; Drost et al., 1991; Kirchner et al., 2013). Our results also suggested that NAC probably had to be transported into the neutrophil cell because its effect was observed after extensive cell washing. Additionally, neutrophils can exert long-term effects even after short time of incubation with NAC (Sadowska et al., 2006; Stolarek et al., 2002), a report that confirms our observation. As a further consequence, NAC can inhibit the activity of PHOX or other neutrophil enzymes such as MPO (Zafarullah et al., 2003; Kharazmi et al., 1988; Drost et al., 1991), leading to a significant decrease in DNA release into extracellular space. Such the effect was also presented by Kirchner et al. (2013) but at a slightly higher dose of the drug than its expected concentration in serum. Our detailed examination of NAC effects on the ability of neutrophils to release NETs showed that this drug acted directly on the ROS-dependent netosis pathway, as neutrophils pretreated with therapeutic concentration of NAC did not release NETs in response to PMA treatment, in contrast to the stimulation with $0.03 \% \mathrm{H}_{2} \mathrm{O}_{2}$. Moreover, ROS-independent netosis pathway was not susceptible to the drug action. Referring to the main applications of NAC as a pulmonary drug, the described results suggest an important role of NAC in respiratory disease treatment. NET release can serve as an efficient antimicrobial weapon of the host, but had also negative consequences, such as increasing viscosity of mucus, e.g., in cystic fibrosis, or the intensification of alveoli damages by NET-bound proteolytic enzymes or released ROS. However, neutrophils often release NETs in lungs as a sterile inflammation, and in this case the local application of NAC seems to be a very interesting proposal for acute and chronic lung disease treatment, where inhibition of NET production may protect the alveoli walls from damages.

Ethamsylate belongs to the cardiovascular drugs, commonly used in a massive hemorrhage, bleeding, pro- longed menstruation or vascular purpura (El-Shabrawy et al., 2004). This non-thrombogenic hemostatic agent acts on the vascular wall, restoring capillary resistance. However, the mechanism of its action is still poor understood. It is known that ethamsylate exerts an effect on platelet aggregation (Alvarez-Guerra et al., 2002; Hernandez et al., 2004). On the other hand, activated platelets induce the formation of NETs in transfusionrelated acute lung injury (Caudrillier et al., 2012). There are no reports about the impact of ethamsylate on the activity and function of neutrophils, although the drug is known to activate lymphocytes and inhibit macrophages (Dayrens et al., 1983). Our study presented for the first time that neutrophil treatment with ethamsylate caused a significant, dose-dependent decrease of ROS production. Even a short incubation of neutrophils in presence of this drug was sufficient to reduce the amount of ROS. Sack and Ceruttil (1973) showed that ethamsylate bounds to the cell surface and changes the net membrane surface charge, a finding that could explain the rapid response of neutrophils to ethamsylate action.

The reduction of NET formation by neutrophils, treated with ethamsylate seems to be clear as regards the ROS inhibition. However, the precise examination of possible influence of the drug on neutrophils presented that the ROS-independent pathway was also the target for ethamsylate, or that the drug can influence the common part of both netosis pathways. To explain the exact action of this drug, further studies are needed.

However, treatment of bleeding with ethamsylate can decrease efficiency of clot formation in blood vessels, resulting from inhibited netosis. Moreover, ethamsylate seems to act as a double-edged sword, on one hand increasing a tightness of the blood vessels but, on the other, limiting the possibility of clot formation. Application of this drug may be interesting as a support therapy in vein thrombosis or myocardial infarction, where the restriction of ability to clot formation is needed.

Ketoprofen is a non-steroidal, anti-inflammatory drug (NSAID) commonly used for treatment of pain, fever and inflammation (Costa et al., 2006). However, the impact of ketoprofen or other NSAIDs on netosis has never been examined. At low doses, ketoprofen inhibits the synthesis of prostaglandins, but for anti-inflammatory action higher doses are required (Costa et al., 2006), at which the drug inhibits the oxidative burst of neutrophils. As the effectiveness of low-dose treatment with ketoprofen was still under discussion (Benbarek et al., 2012; Costa et al., 2006) we performed the analysis of its influence on ROS production at the a wide range of drug concentration and clearly observed lowering ROS production by ketoprofen-pretreated neutrophils at the whole concentration range tested. Surprisingly, at the same time the NET production increased significantly. A detailed examination of the influence of ketoprofen treatment on netosis pointed out on the activation of ROS-independent pathway, reaffirming the anti-inflammatory properties of this drug. However, the details of the mechanism of its action are still not clear and need more extended studies.

Clemastine, that modulates the activation and chemotaxis of immune cells (Cíž \& Lojek, 2013), did not presented any significant influence on either ROS production or NET generation at therapeutic doses. Some impact on PMA-activated ROS production was detected only at clemastine concentration that 3 -fold exceeds the medically applied dose.

The effect of hydrocortisone on neutrophils has been repeatedly studied, and showed the inhibition of phago- 
Table 1. Effect of selected drugs on neutrophils

\begin{tabular}{|c|c|c|c|}
\hline Drug & Effect on viability & Effect on ROS production & Effect on NET formation \\
\hline $\mathrm{N}$-acetylcysteine & No effect & Inhibition in dose-dependent manner & Inhibition of ROS-dependent pathway \\
\hline Ketoprofen & No effect & Inhibition in dose-dependent manner & $\begin{array}{l}\text { Activation of netosis pathway (probably ROS-inde- } \\
\text { pendent) }\end{array}$ \\
\hline Ethamsylate & Increased & Inhibition in dose-dependent manner & $\begin{array}{l}\text { Inhibition of ROS-dependent and ROS-independent } \\
\text { pathways }\end{array}$ \\
\hline Clemastine & No effect & No effect & No effect \\
\hline Hydrocortisone & No effect & No effect & No effect \\
\hline
\end{tabular}

cytosis (Petroni et al., 1988) and degranulation (Coates et al., 1983), as well as impaired chemotaxis (Ward, 1966). Moreover, it was presented that hydrocortisone, at high dosage, efficiently inhibited the oxidative burst of neutrophils in vivo and in vitro (Dandona et al., 2001, 1999, 1998). However, our results, in which the medically applicable doses were used, did not present any effects of hydrocortisone on oxidative or not-oxidative pathways of NET formation.

All presented results are summarized in Table 1 and can be helpful in the consideration of the medical application of analyzed drugs where the possible impact on netosis processes may influence the progression of the disease or its treatment.

\section{Competing Interests}

The authors declare that they have no competing interests.

\section{Acknowledgements}

This work was supported by the National Science Centre, Poland (grant No. 2012/05/B/NZ1/00003 to MRK).

\section{REFERENCES}

Alvarez-Guerra M, Hernandez MR, Escolar G, Chiavaroli C, Garay RP, Hannaert P (2002) The hemostatic agent ethamsylate enhances P-selectin membrane expression in human platelets and cultured endothelial cells. Thromb Res 107: 329-335.

Amulic B, Cazalet C, Hayes GL, Metzler KD Zychlinsky A (2012) Neutrophil function: from mechanisms to disease. Annu Rev Immunol 30: 459-489.

Annane D, Sébille V, Charpentier C, Bollaert PE, François B, Korach JM, Capellier G, Cohen Y, Azoulay E, Troché G (2002) Effect of treatment with low doses of hydrocortisone and fludrocortisone on mortality in patients with septic shock. IAMA 288: 862-871.

Aruoma OI, Halliwell B, Hoey BM, Butler J (1989) The antioxidant action of $\mathrm{N}$-acetylcysteine: its reaction with hydrogen peroxide, hydroxyl radical, superoxide, and hypochlorous acid. Free Radic Biol Med 6: 593-597.

Benbarek H, Ayad A, Deby-Dupont G, Boukraa L, Serteyn D (2012) Modulatory effects of non-steroidal anti-inflammatory drugs on the luminol and lucigenin amplified chemiluminescence of equine neutrophils. Vet Res Commun 36: 29-33.

Bianchi M, Hakkim A, Brinkmann V, Siler U, Seger R, Zychlinsky A, Reichenbach, J (2009) Restoration of NET formation by gene therapy in CGD controls aspergillosis. Blood 114: 2619-2622.

Bjornson BH, Harvey JM, Rose L (1985) Differential effect of hydrocortisone on eosinophil and neutrophil proliferation. J Clin Invest 76: 924-929.

Borgström L, Kågedal B, Paulsen O (1986) Pharmacokinetics of Nacetylcysteine in man. Eur J Clin Pharmacol 31: 217-222.

Brinkmann V, Reichard U, Goosmann C, Fauler B, Uhlemann Y, Weiss DS, Weinrauch Y, Zychlinsky A (2004) Neutrophil extracellular traps kill bacteria. Science 303: 1532-1535.

Brinkmann V, Zychlinsky A (2012) Neutrophil extracellular traps: is immunity the second function of chromatin? I Cell Biol 198: 773 783.
Caudrillier A, Kessenbrock K, Gilliss BM, Nguyen JX, Marques MB, Monestier M, Looney MR (2012) Platelets induce neutrophil extracellular traps in transfusion-related acute lung injury. I Clin Invest 122: 2661-2671.

Chow O, Von Köckritz-Blickwede M, Bright T, Hensler ME, Zinkernagel AS, Cogen AL, Nizet V (2010) Statins enhance formation of phagocyte extracellular traps. Cell Host and Microbe 8: 445-454.

Cíž M, Lojek A (2013) Modulation of neutrophil oxidative burst via histamine receptors. Br I Pharmacol 170: 17-22.

Coates TD, Wolach B, Tzeng DY, Higgins C, Baehner RL, Boxer LA (1983) The mechanism of action of the antiinflammatory agents dexamethasone and Auranofin in human polymorphonuclear leukocytes. Blood 62: 1070-1077.

Costa D, Moutinho L, Lima JLFC, Fernandes E (2006) Antioxidant activity and inhibition of human neutrophil oxidative burst mediated by arylpropionic acid non-steroidal anti-inflammatory drugs. Biol Pharm Bull 29: 1659-1670.

Dale DC, Fauci AS, Guerry D, Wolff SM (1975) Comparison of agents producing a neutrophilic leukocytosis in man Hydrocortisone, prednisone, endotoxin, and etiocholanolone. J Clin Invest 56: 808-813.

Dandona P, Aljada A, Ghanim H, Mohanty P, Hamouda W, AlHaddad W (2001) Acute suppressive effect of hydrocortisone on p47 subunit of nicotinamide adenine dinucleotide phosphate oxidase. Metabolism 50: 548-552.

Dandona P, Suri M, Hamouda W, Aljada A, Kumbkarni Y, Thusu K (1999) Hydrocortisone-induced inhibition of reactive oxygen species by polymorphonuclear neutrophils. Crit Care Med 27: 2442-2444.

Dandona P, Thusu K, Hafeez R, Abdel-Rahman E, Chaudhuri A (1998) Effect of hydrocortisone on oxygen free radical generation by mononuclear cells. Metabolism 47: 788-791.

Dayrens P, Ivanoff B, Cussac M, Fontanges R (1983) Antiinflammatory and immunostimulant activities of six sulphur compounds-four benzenesulphonates, levamisole, and pyritinol hydrochloride-assayed in mouse cell activation studies. Arzneimittelforschung 33: 372-377.

Douda DN, Khan M, Grasemann H, Palaniyar N (2015) SK3 channel and mitochondrial ROS mediate NADPH oxidase-independent NETosis induced by calcium influx. Proc Natl Acad Sci 112: 2817-2822.

Drost E, Lannan S, Bridgeman MME, Brown D, Selby C, Donaldson K, MacNee W (1991) Lack of effect of N-acetylcysteine on the release of oxygen radicals from neutrophils and alveolar macrophages. Eur Respir J 4: 723-729.

Duranton J, Belorgey D, Carrère J, Donato L, Moritz T, Bieth JG (2000) Effect of DNase on the activity of neutrophil elastase, cathepsin $G$ and proteinase 3 in the presence of DNA. FEBS Lett 473: 154-156.

El-Shabrawy Y, El-Enany N, Salem K (2004) Sensitive kinetic spectrophotometric determination of captopril and ethamsylate in pharmaceutical preparations and biological fluids. Farmaco 59: 803-808.

Fuchs TA, Abed U, Goosmann C, Hurwitz R, Schulze I, Wahn V, Weinrauch Y, Brinkmann V, Zychlinsky A (2007) Novel cell death program leads to neutrophil extracellular traps. J Cell Biol 176: $231-$ 241.

Fuchs TA, Brill A, Duerschmied D, Schatzberg D, Monestier M, Myers DD, Wagner D D (2010) Extracellular DNA traps promote thrombosis. Proc Natl Acad Sci USA 107: 15880-15885.

Fuchs TA, Brill A, Wagner DD (2012) Neutrophil extracellular trap (NET) impact on deep vein thrombosis. Arteriosclerosis, Thrombosis, and Vascular Biology 32: 1777-1783.

Gerilechaogetu, Hanieh H, Abe A, Kondo Y (2009) Extracellular signal-regulated kinase (ERK) activation in chicken heterophils stimulated with phorbol 12-myristate 13-acetate (PMA), formyl-methionylleucyl-phenylalanine (fMLP) and lipopolysaccharide (LPS). Anim Sci J 80: 577-584.

Guimarães-Costa AB, Nascimento MTC, Wardini AB, Pinto-da-Silva LH, Saraiva E M (2012) ETosis: a microbicidal mechanism beyond cell death. I Parasitol Res 2012: 929743.

Gupta AK, Giaglis S, Hasler P, Hahn S (2014) Efficient neutrophil extracellular trap induction requires mobilization of both intracellular 
and extracellular calcium pools and is modulated by cyclosporine A. PLOS ONE 9: 19-21.

Hakkim A, Fuchs T, Martinez NE, Hess S, Prinz H, Zychlinsky A, Waldmann $H$ (2011) Activation of the Raf-MEK-ERK pathway is required for neutrophil extracellular trap formation. Nat Chem Biol 7: 75-77.

Hernandez MR, Alvarez-Guerra M, Escolar G, Chiavaroli C, Hannaert P, Garay, RP (2004) The hemostatic agent ethamsylate promotes platelet/leukocyte aggregate formation in a model of vascular injury. Fundam Clin Pharmacol 18: 423-430.

Heyn J, Beiras-Fernandez A, Luchting B, Briegel J, Weis F (2011) Inflammatory reactions and hydrocortisone in the setting of cardiac surgery: an overview. Cardiovasc Hematol Agents Med Chem 9: 56-61.

Holdiness, MR (1991) Clinical pharmacokinetics of $\mathrm{N}$-acetylcysteine. Clin Pharmacokinet 20: 123-134.

Kaplan MJ, Radic M (2012) Neutrophil extracellular traps: doubleedged swords of innate immunity. J Immunol 189: 2689-2695.

Keshari RS, Verma A, Barthwal MK, Dikshit M (2012) Reactive oxygen species-induced activation of ERK and p38 MAPK mediates PMA-induced NETs release from human neutrophils. I Cell Biochem 15: $1-24$.

Kharazmi A, Nielsen H, Schiøtz PO (1988) N-acetylcysteine inhibits human neutrophil and monocyte chemotaxis and oxidative metabolism. Int J Immunopharmacol 10: 39-46.

Kirchner T, Hermann E, Möller S, Klinger M, Solbach W, Laskay T, Behnen M (2013) Flavonoids and 5-aminosalicylic acid inhibit the formation of neutrophil extracellular traps. Mediators Inflamm 2013: $1-10$.

Kumar V, Sharma A (2010) Neutrophils: Cinderella of innate immune system. Int Immunopharmacol 10: 1325-1334.

Lapponi MJ, Carestia A, Landoni VI, Rivadeneyra L, Etulain J, Negrotto S, Pozner RG, Schattner M (2013) Regulation of neutrophil extracellular trap formation by anti-inflammatory drugs. $J$ Pharmacol Exp Ther 345: 430-437.

Li Y, Trush MA (1998) Diphenyleneiodonium, an NAD(P)H oxidase inhibitor, also potently inhibits mitochondrial reactive oxygen species production. Biochem Biophys Res Commun 253: 295-299.

Liles WC, Dale DC, Klebanoff SJ (1995) Glucocorticoids inhibit apoptosis of human neutrophils. Blood 86: 3181-3188.

Lojek A, Cíž M, Pekarová M, Ambrožová G, Vašíček O, Moravcová J, Kubala L, Drábiková K, Jančinová V, Perečko T (2011) Modulation of metabolic activity of phagocytes by antihistamines. Interdiscip Toxicol 4: 15-19.

Marik PE, Pastores SM, Annane D, Meduri GU, Sprung CL, Arlt W, Keh D, Briegel J, Beishuizen A, Dimopoulou I (2008) Recommendations for the diagnosis and management of corticosteroid insufficiency in critically ill adult patients: consensus statements from an international task force by the American College of Critical Care Medicine. Critical Care Med 1937-1949.

Masha A, Martina V (2014) Endothelial dysfunction in metabolic diseases: role of oxidation and possible therapeutic employment of $\mathrm{N}$ acetylcysteine. Curr Med Chem 32: 3616-3635.

McLeish KR, Merchant ML, Klein JB, Ward RA. (2013) Technical note: proteomic approaches to fundamental guestions about neutrophil biology. J Leucocyte Biol 94: 682-692.

Nanì S, Fumagalli L, Sinha U, Kamen L, Scapini P, Berton, G (2015) Src family kinases and syk are required for neutrophil extracellular trap formation in response to $\beta$-glucan particles. J Innate Immun 7: 59-73.

Nishinaka Y, Arai T, Adachi S, Takaori-Kondo A, Yamashita K (2011) Singlet oxygen is essential for neutrophil extracellular trap formation. Biochem Biophys Res Commun 413: 75-79.

Nosál R, Drábiková K, Číž M, Lojek A, Danihelová E (2002) Effect of H1-antagonist Dithiaden ${ }^{\circledR}$ on human PMN-leukocyte aggregation and chemiluminescence is stimulus-dependent. Inflamm Res 51: $557-562$.

Papayannopoulos V, Staab D, Zychlinsky A (2011) Neutrophil elastase enhances sputum solubilization in cystic fibrosis patients receiving DNase therapy. Plos One 6: e28526.
Parker H, Dragunow M, Hampton MB, Kettle J, Winterbourn CC (2012) Requirements for NADPH oxidase and myeloperoxidase in neutrophil extracellular trap formation differ depending on the stimulus. I Lenkoc Biol 92: 841-849.

Petroni KC, Shen L, Guyre PM (1988) Modulation of human polymorphonuclear leukocyte IgG Fc receptors and Fc receptor-mediated functions by IFN- $\gamma$ and glucocorticoids. I Immunol 140: 3467-3472.

Pilsczek FH, Salina D, Poon KKH, Fahey C, Yipp BG, Sibley CD, Robbins SM, Green FHY, Surette MG, Sugai M (2010) A novel mechanism of rapid nuclear neutrophil extracellular trap formation in response to Staphylococcus aureus. J Immunol 185: 7413-7425.

Regenthal R, Krueger M, Koeppel C, Preiss R (1999) Drug levels: therapeutic and toxic serum/plasma concentrations of common drugs. $J$ Clin Monit Comput 15: 529-544.

Remijsen Q, Kuijpers TW, Wirawan E, Lippens S, Vandenabeele P, Vanden Berghe T (2011) Dying for a cause: NETosis, mechanisms behind an antimicrobial cell death modality. Cell Death Differ 18: $581-588$.

Sack ES, Cerutti N (1973) Effects of cyclonamine on blood platelets II Changes in the surface charge and inhibition of the release reaction. Medicina (B Aires) 33: 685-694.

Sadowska, A M, Manuel-Y-Keenoy, B, Vertongen, T, Schippers, G, Radomska-Lesniewska, D, Heytens, E, and De Backer, W a (2006) Effect of $\mathrm{N}$-acetylcysteine on neutrophil activation markers in healthy volunteers: In vivo and in vitro study. Pharmacol Res 53: 216-225.

Saffarzadeh M, Juenemann C, Queisser M, Lochnit G, Barreto G, Galuska SP, Preissner KT (2012) Neutrophil extracellular traps directly induce epithelial and endothelial cell death: a predominant role of histones. PloS One 7: e32366

Schleimer RP, Freeland HS, Peters SP, Brown KE, Derse CP (1989) An assessment of the effects of glucocorticoids on degranulation, chemotaxis, binding to vascular endothelium and formation of leukotriene B4 by purified human neutrophils. J Pharmacol Exp Ther 250: 598-605.

Sprung CL, Annane D, Keh D, Moreno R, Singer M, Freivogel K, Weiss YG, Benbenishty J, Kalenka A, Forst H, (2008) Hydrocortisone therapy for patients with septic shock. N Engl J Med 358: 111-124.

Stolarek R, Białasiewicz P, Nowak D (2002) N-acetylcysteine effect on the luminol-dependent chemiluminescence pattern of reactive oxygen species generation by human polymorphonuclear leukocytes. Pulm Pharmacol Ther 15: 385-392.

Taniguchi K, Masuda Y, Takanaka K (1991) Inhibitory effects of histamine $\mathrm{H} 1$ receptor blocking drugs on metabolic activations of neutrophils. J Pharmacobiodyn 14: 87-93.

Urban CF, Ermert D, Schmid M, Abu-Abed U, Goosmann C, Nacken W, Brinkmann V, Jungblut PR, Zychlinsky A (2009) Neutrophil extracellular traps contain calprotectin, a cytosolic protein complex involved in host defense against Candida albicans. PLoS Pathog $\mathbf{5}$.

Ward, PA (1966) The chemosuppression of chemotaxis. J Exp Med 124: 209-226.

Weyts FAA, Flik G, Verburg-Van Kemenade, BML (1998) Cortisol inhibits apoptosis in carp neutrophilic granulocytes. Dev Comp Immunol 22: $563-572$

Wozniok I, Hornbach A, Schmitt C, Frosch M, Einsele H, Hube B, Löffler J, Kurzai O (2008) Induction of ERK-kinase signalling triggers morphotype-specific killing of Candida albicans filaments by human neutrophils. Cell Microbiol 10: 807-820.

Wu XY, Luo AY, Zhou YR, Ren JH (2014) N-acetylcysteine reduces oxidative stress, nuclear factor $x \mathrm{~B}$ activity and cardiomyocyte apoptosis in heart failure. Mol Med Rep 10: 615-624.

Zafarullah M, Li WQ, Sylvester J, Ahmad M (2003) Molecular mechanisms of N-acetylcysteine actions. Cell Mol Life Sci 60: 6-20.

Zawrotniak M, Rapala-Kozik M (2013) Neutrophil extracellular traps (NETs) formation and implications. Acta Biochim Pol 60: 277-284. 